\title{
Research of Automatic Recognition Double Layers Intrusion Intention Algorithm Based on Attack Graph
}

\author{
Wang Guangze, Wang Peng, Luo Zhiyong, Zhu Suxia \\ Harbin University of Science and Technology, Harbin 150080, Heilongjiang \\ Province, China \\ wangguangze_hust@sina.com
}

\begin{abstract}
The determination of network equipment weaknesses and the discovery of intrusion intention is one of the difficulties that troubled network security management personnel. Based on previous studies, further proposed a double attack graph based on domainequipment. By the underlying network topology data collected and analyzed, using Bayesian theory to complete the quantify for the double attack graph and generation strategy in minimal power key set, with the cost of calculation of key equipment in the automatic recognition network topology, we provide an important basis for network maintenance. Experimental results show that the measure of using quantitative domainequipment double attack graph to recognize the intrusion intention is not only effective and feasible, but also has the feature of easy promotion.
\end{abstract}

Keywords: network security; intrusion intention; attack graph; recognition algorithm

\section{Introduction}

Intrusion intention identification refers to a process in the Internet environment, through the analysis of a large number of feedback alarm data from the underlying intrusion detection system, and effectively judging the means of the invaders and the ultimate goal, which is the essence of the scientific analysis of the intrusion process[1 3]. The fast and effective method of identifying intrusion intention provide an important basis for network management personnel to better maintain network security, it is also the premise for early detection and prevention by the network security threats, an important part of network security situation analysis, and the emerging field of network security research focus.

In the computer field, artificial intelligence achieved the recognition of intention earliest. Bratman $\mathrm{M}$ used the recognition of intention for decision-making and action planning [4]. Swiler, who first introduced the attack graph, using the nodes and edges of graph to formalize association between network devices, providing a new approach [5] to identify intrusion intention. Sheyner, who used the greedy algorithm to optimize the attack graph, identifying the key weaknesses in the minimum set throughout network, and as a theoretical basis for the recognition of intrusion intention [6]. Ou X further improved the thoughts of attack graph, putting forward a fast method to identify intrusion intention [7]. Lippmann and others increased constraints on the attack graph nodes and weakness, thereby reducing the attack graph scale, providing a quick method to quantify [8]. Roschek used scanning tools NESSUS to find the key nodes in the network, generating a key node attack graph by the algorithm that search through the front and rear, effectively prevent the intrusion occurring [9]. Ye further quantified the attack graph by the probability of intrusion occurred to provide a theoretical basis [10] to identify intrusion intention. Chen Xiaojun, who proposed the idea of the probability and statistics to apply to the attack graph, the attack graph quantified provided the basis to further determine the intention of the network, effectively preventing the intrusion [11]. 
On the basis of years of research [12], the group further proposed the double attack graph model based on domain-equipment, and using Bayesian probability theory to carry out the research about the automatic recognition of intrusion intention.

\section{Building Automatic Recognition Model for Intrusion Intention}

\subsection{Intrusion Intention and Recognition Model}

Intrusion intention is an intruder original purpose, and it is expressed as an intruder brings the damage for the network which is invaded. Intrusion intention recognition is a process that is the network security people taking measures for the invaders intent which is expresses as forecast, analysis, evaluation and block. Its recognition model structure is shown in Figure 1.

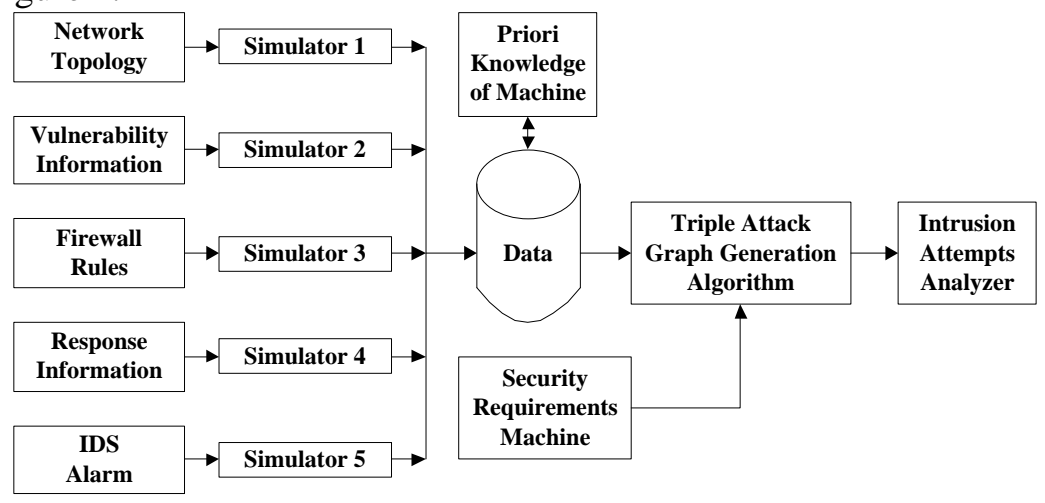

Figure 1. Architecture Diagram of Intrusion Intent Recognition Model

\subsection{Domain-equipment Two-tier Attack Graph}

To create a domain-equipment double attack graph model, we formalized the following constraints:

$W$ represents a collection of weakness in the network device, then for any one weakness $w$ in set $W, w$ can be defined as a triple $\left(w_{i d}, w_{p}, w_{c}\right)$. In the tuple, wid is the number of the weakness in the network security CVE standard library; $w_{p}$ is the set of precursor conditions that intruder can successfully exploit this vulnerability; $w_{c}$ is the set of harm that an intruder using the vulnerability cause on the network.

$I P$ represents the set of the equipment in network layer, then for any one network equipment ip in $I P$, ip defined as triples $(W, o, N e t I P)$. In the triple, $W$ is the set of network equipment weaknesses; $o$ is the set of open ports in network equipment; NetIP is the set of other devices connected to the network.

$D$ represents the set of network topology domains, then for any one domain $d$ in set $D$, $d$ may be defined as triples $(I P, N e t D)$. In the triple, $I P$ is a set of network equipment in the domain; NetD is a set of other domains connected to the domain.

$E$ represents the set of node equipment, then for any one node device $e$ in set $E, e$ may be defined as a triples $\left(e_{i d}, D, I P\right)$. In the triple, $e_{i d}$ is the node number of the device; $D$ is the set of the node equipment in the domain; $I P$ is a set of network equipment that belongs to the node equipment.

In the daily intrusion events, the intruder first scans all the weaknesses of a node equipment $\mathrm{e}$ in the network topology, forming weakness set $w$. By analyzing the set $w$, the intruders may invade the ip of equipment in some domain $d$, completing the controlling process that make the privilege of $i p$ change from low to high. Secondly, by the set NetIP of device $i p$, invading other equipment $i p^{\prime}$ in the domain $d$, the intruders completely control the domain d step by step eventually. Finally, by the set NetD of domain $d$, the intruders continue invading other domains $d^{\prime}$ in the same way, realizing their invasion 
intention eventually. Thus, the domain-equipment double attack graph model shown in Figure 2.

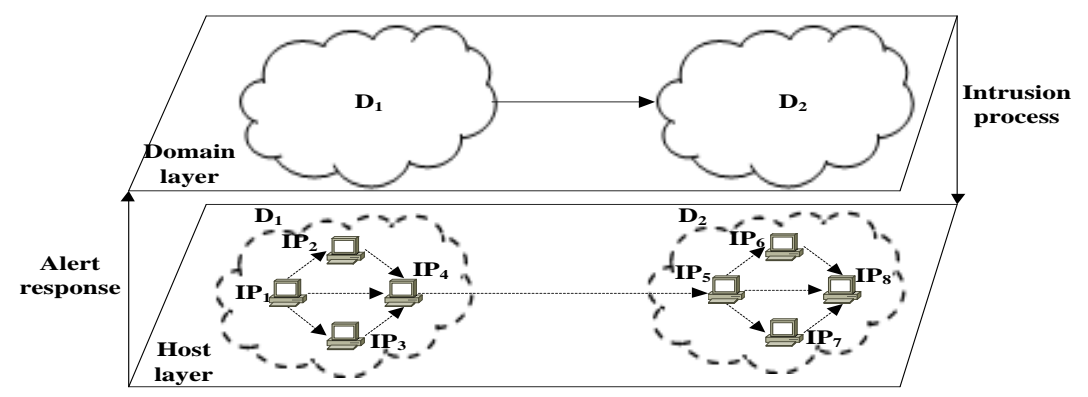

Figure 2. Domain-equipment Double Attack Graph Model

Definition 1: domain-equipment double attack graph $V$, it is a view and formalized as a triples $\left(V_{D}\left(E_{d}, L_{d}\right), V_{I P}\left(E_{i p}, L_{i p}\right)\right)$, where: $V_{D}\left(E_{d}, L_{d}\right)$ is the domain layer attack graph; $V_{I P}$ $\left(E_{i p}, L_{i p}\right)$ is the equipment layer attack graph; $E_{q}$ is a set of node equipment; $L_{q}$ is the set of the routes between the node equipment; $q$ is $d$ or $i p$. In the definition 1, if there is a route that make node equipment $e_{i}$ turn to the node equipment $e_{j}$, it means the intruders invade the node equipment $e_{j}$ by the node equipment $e_{i}$ successfully, where: $l_{i j} \in L_{q}, e_{i} \in E_{q}, e_{j} \in E_{q}$, $e_{i} \neq e_{j}, q=d$ or $i p$.

\subsection{Attack Graph Generation Strategy}

Domain-equipment two-tier attack graph $V$ generation policy obeys the following restrictions:

(1) If an intruder has gained a network equipment elevated privileges, and the intruder is no longer regain the network equipment lower privileges.

(2) If the intruder has successfully invaded network equipment, the intruder is no longer re-invasion of the network equipment.

(3) In the process of an intruder achieving his intention invasion, his invasion operations are necessary and non-redundant.

(4) An intruder gets a network equipment's permission from low to high, and the orders are Null, Guest, Admin.

Generation strategy 1 of the domain-equipment double attack graph follows:

Step1: Initialize policy-related variables, and set Pow for permission variable;

Step2: Remove a equipment from the set $I P$ of network equipment in device layer to store in variable ip;

Step3: Set permission variable Pow corresponding the variable ip empty Null, computing the rank of the set of the weakness in network equipment and assigning the variable with Num;

Step4: Make the loop variable $i=1$;

Step5: Make loop variable $j=i+1$;

Step6: If $w_{c}$ that the weakness $w_{i}$ was successfully invaded lead to meet the precondition $w_{p}$ that weakness $w_{j}$ be invaded, namely satisfy the relationship $w_{j} \times w_{p} \subseteq w_{i}$ $\times w_{c}$, then assigning the permission variable Pow corresponding variable ip with Guest or Admin and perform Step9, otherwise perform Step7;

Step7: Let the variable $j=j+1$, determine $j$ whether the variable Num equal, if not perform Step6, otherwise perform Step8;

Step8: Let the variable $\mathrm{i}=\mathrm{i}+1$, judge $\mathrm{i}$ whether the Num-1 equal to, if not perform Step5, otherwise perform Step9;

Step9: Remove the next device from the set $I P$ of the network equipment in device layer to store in the variable $i p$;

Step10: If the set $I P$ is not empty, then perform Step3, and otherwise perform Step11; 
Step11: Recover the set IP of the network equipment in device layer and set Num to the rank of set $I P$;

Step12: Make the loop variable $i=1$;

Step13: Make loop variable $j=i+1$;

Step14: If the permission of the equipment $i p_{i}$ is not null, namely the Pow corresponding equipment $i p_{i}$ is Guest or Admin, then perform Step15, otherwise perform Step17;

Step15: If the equipment $i p_{i}$ and $i p_{j}$ can be connected through port $o$ and it can invade equipment $i p_{j}$ via the equipment $i p_{i}$ and access the privilege Guest or Admin of the equipment $i p_{j}$, then perform Step16, otherwise perform Step17;

Step16: add the equipment $i p_{i}$ and $i p_{j}$ to equipment attack graph $\operatorname{VIP}\left(E_{i p}\right)$,and add the edge $i p_{i} \rightarrow i p_{j}$ to the attack graph VIP $\left(L_{i p}\right)$ in the device layer;

Step17: Let the variable $j=j+1$, determine $j$ whether the variable Num equal to, if not perform Step14, otherwise perform Step18;

Step18: Let the variable $i=i+1$, judge $i$ whether the Num-1 equal to, if not perform Step13, otherwise perform Step19;

Step19: Add the attack graph $\operatorname{VIP}\left(E_{i p}, L_{i p}\right)$ in device layer to the double attack graph $V$;

Step20: Make the loop variable $i=1$;

Step21: Make loop variable $j=i+1$;

Step22: Make $d_{1}$ as the protection domain for equipment $i p_{i}, d_{2}$ as the protection domain for equipment $i p_{j}$;

Step23: If the two domains are not same, that $d_{1}\left\langle>d_{2}\right.$, and the equipment $i p_{i}$ has been invaded, then perform Step24, otherwise perform Step26;

Step24: Add the protection domain of the equipment $i p_{i}$ to domain layer attack graph $V D\left(E_{d}\right)$

Step25: If it can invade equipment $i p_{j}$ through port $o$ in equipment $i p_{i}$ and improve the privilege of the control equipment $i p_{j}$ to Guest or Admin, then add the protection domain of the equipment $i p_{j}$ to the domain layer attack graph $V D\left(E_{d}\right)$, add the edge $d\left(i p_{i}\right) \rightarrow d\left(i p_{j}\right)$ to the domain layer attack graph $V D\left(L_{d}\right)$, otherwise perform Step26;

Step26: Let the variable $j=j+1$, determine $j$ whether the variable Num equal to, if not perform Step22, otherwise perform Step27;

Step27: Let the variable $i=i+1$, judge $i$ whether the Num-1 equal to, if not perform Step21, otherwise perform Step28;

Step28: Add the domain layer attack graph $V D\left(E_{d}, L_{d}\right)$ to the double attack graph $V$.

After analysis, the time complexity of the policy 1 is $O\left(|I P| \times\left|W_{i p}\right|^{2}\right)$.

\section{Quantitative Analysis of Intrusion Intention and Response Strategies}

\subsection{Quantitative Analysis of Intrusion Intention}

In the double attack graph of the domain-equipment, the property of weakness determines each node that intruder can invade successfully. Therefore, this article will divide the property of the device weakness into three parts: easiness $\alpha$, privacy $\beta$ and the rate of return $\gamma$. Depending on the complexity of the actual network operations, the author made assessment and assignment with each property of the weakness, as shown in Table 1 , the probability of an intruder successfully exploited weaknesses $\mathrm{w}$ as follows:

$$
\pi\left(\begin{array}{lll}
w & =
\end{array} \quad \begin{array}{lll}
\delta_{1} \alpha & +\delta_{2} \beta & +\delta_{3} \gamma
\end{array}\right.
$$

Where: $\delta_{1}, \delta_{2}, \delta_{3}$ are weights assigned by the network management personnel in accordance with the actual situation. 


\section{Table 1. Properties and Assignment of the Weakness}

\begin{tabular}{ccc}
\hline Property & Degree & Assignment \\
\hline \multirow{3}{*}{$\alpha$} & easy & 0.9 \\
& secondary & 0.6 \\
& difficult & 0.3 \\
$\beta$ & low & 0.6 \\
& mid & 0.8 \\
& high & 0.9 \\
$\gamma$ & low & 0.5 \\
& mid & 0.7 \\
& high & 0.9 \\
\hline
\end{tabular}

Definition 2: Invasion route. In the domain-equipment double attack graph $V$, if there exits the set $E^{\prime}$ of node equipment, it may make the intruder start from node equipment $e_{0}$ along with the equipment in set $E^{\prime}$ to realize the intrusion intention, then the node equipment set $E^{\prime}$ and the link of them in domain-equipment double attack graph called a intrusion route, denoted by $r$. In the attack graph $V$, all the intrusion paths constitute a collection that is called invasion path set, denoted $R$.

In the attack graph of equipment level, if there exits $j$ invasion path through $k$ node equipment, and the intruder can achieve its intrusion intention $i$, then the probability of intrusion intention $i$ that may happen as follows:

$$
\pi(i)=1-\prod_{j}\left[1-\prod_{k} \pi\left(\pi_{k}\right)\right]
$$

By Bayesian formula, we can calculate the relative probability of an intruder may realize the intrusion intention by intrusion route $t$ :

$$
\pi\left(i p_{t} \mid i\right)=\frac{\pi\left(i \mid i p_{t}\right) \times \pi\left(i p_{t}\right)}{\pi(i)}, t=1,2, \ldots, j
$$

If the relative probability of some invasion path is higher, it indicates the possibility of an intruder from the invasion path to achieve its intentions may be high. For this reason, network managers should focus on protecting the node equipment through the invasion route.

\subsection{Minimal Right Key Set of Attack Graph}

Definition 3: Minimal key right set. In the domain-equipment double attack graph $V$, $V(E)$ is the set of node equipment, it makes $K_{i}$ the nonempty set that does not contain the start node and the destination node and meet $K_{i} \subset V(E)$, if any intrusion route $r$ in set $R$ through all node equipment in $K_{i}$, there call $K_{i}$ is the minimal key right set $K_{\min w}$.

For the generation strategy to design minimal right key set $K_{\min W}$, this paper follows the formal constraint:

$A_{\text {rank }}$ represents the rank of the node equipment set $V(E)$, there: $A_{\text {rank }}=|V(E)| ; D_{\text {rank }}$ represents the rank of the intrusion route set $R$, there: $D_{\text {rank }}=|R|$, and $D_{\text {rank }}=C_{A_{\text {mank }-2}}^{1}+C_{A_{\text {mak }}-2}^{2}+\ldots+C_{A_{\text {tank }}-2}^{A_{\text {ank }}-2} ; r_{i}$ represents any intrusion route, and meet $r_{i} \in R ; E_{i}$ represents the set of all node equipment in the invasion route $r_{i}$; In symbols $r_{i}$ and $E_{i}$, the $i$ $=1,2, \ldots, D_{\text {rank }}$.

The generation strategy of the minimal right key set $K_{\min W}$ in the domain-equipment double attack graph $V$ follows:

Step1: Initialize policy-related variables and set landmark variable Flag is true; 
Step2: Calculate the number of elements in the set $R$ and assign to the variable $i$;

Step3: Set landmark variable Flag is true;

Step4: Calculate the number of elements in the set $V(E)$ and assign to the variable $j$;

Step5: Judge the intersection between the set $K_{i}$ and $K_{j}$ whether empty, if empty then there is a route that not through node in set $K_{i}$, set the landmark variable Flag false and perform Step6; if not empty, then perform Step6;

Step6: Variable $j=j-1$, determine $j$ whether 0 , if 0 perform Step7, otherwise perform Step5;

Step7: Judge the landmark variable Flag whether is true? If true add the set $K_{i}$ to the set $K$;

Step8: Variable $i=i-1$, determine $i$ whether 0, if 0 perform Step9, otherwise perform Step3;

Step9: Find the minimum elements in the set $K$ as the minimum right key set $K_{\min W}$.

After analysis, the time complexity of the strategy 2 is $O\left(D_{\text {rank }} \times A_{\text {rank }}\right)$.

\subsection{Intrusion Intention Response based on the Minimal Right Key Set}

In the double attack graph, the main way is cutting off intrusion route to prevent intrusion intentions. Therefore, the intrusion intention response based on the minimal right key set is economically viable process.

According to the algorithm 2, you can get the minimal right key set $K_{\min W}$ in double attack graph. Suppose $i p_{i}$ is any one node equipment in set $K$, the price of maintaining the node equipment $i p_{i}$ recorded as $\operatorname{Cost}\left(i p_{i}\right)$, including: labor costs, hardware costs and other costs.

Based on the above analysis, the optimal maintenance measures costs of the network managers cut off the intrusion intention follows (4), which the cost of maintain the equipment node in the minimal right key set.

$$
\operatorname{Sum}_{\text {Cost }}=\sum_{i=1}^{\left|K_{\min W}\right|} \operatorname{Cost}\left(i p_{i}\right)
$$

\section{Test and Analysis of the Experiment}

To test the intrusion intention identified algorithm of the domain-equipment double attack graph, experimental environment that research group designed mainly consist of four domains and Internet. Among them, environment field is named: domain $D_{l}$, domain $D_{2}$, domain $D_{3}$ and domain $D M Z$. Access policy for each domain: network device in Domain $D_{1}$ and $D_{2}$ can access each other including the domain $D M Z$; network equipment in domain $D_{3}$ can not access equipment in domain $D M Z$; In domain $D_{1}$ and domain $D_{2}$, network equipment $I P_{4}$ and network equipment $I P_{9}$ can access the database server equipment in domain $D_{3}$; the network equipment $I P_{4}$ in domain $D_{1}$ and the equipment in domain $D_{2}$ can access each other; in their respective domains, network devices can access each other; the inner internet equipment exchange data with Internet through domain $D M Z$; other cross-domain access are disabled. Experimental environment, the weakness of network equipment in the network security CVE standard library and the network topology are shown in Figure 3. 


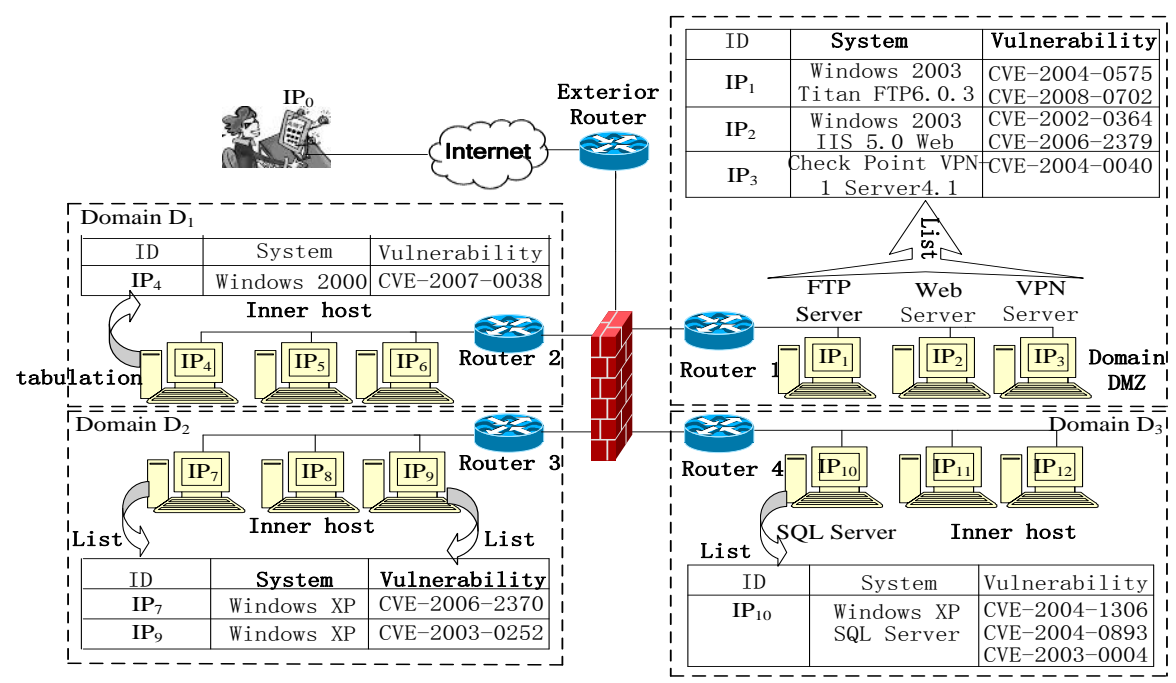

Figure 3. Network Topology and the Weakness of the Equipment

Since the server device $I P_{10}$ stored a lot of sensitive data, so the device is a penetration goal by intruders and the majority of the intrusion refer to, it needs special protection. So the intrusion intention that intruders penetrate network equipment $I P_{10}$ successfully is $i$, then take policy 1 can generate the intrusion intention, the corresponding domainequipment double attack shown in Figure 4.

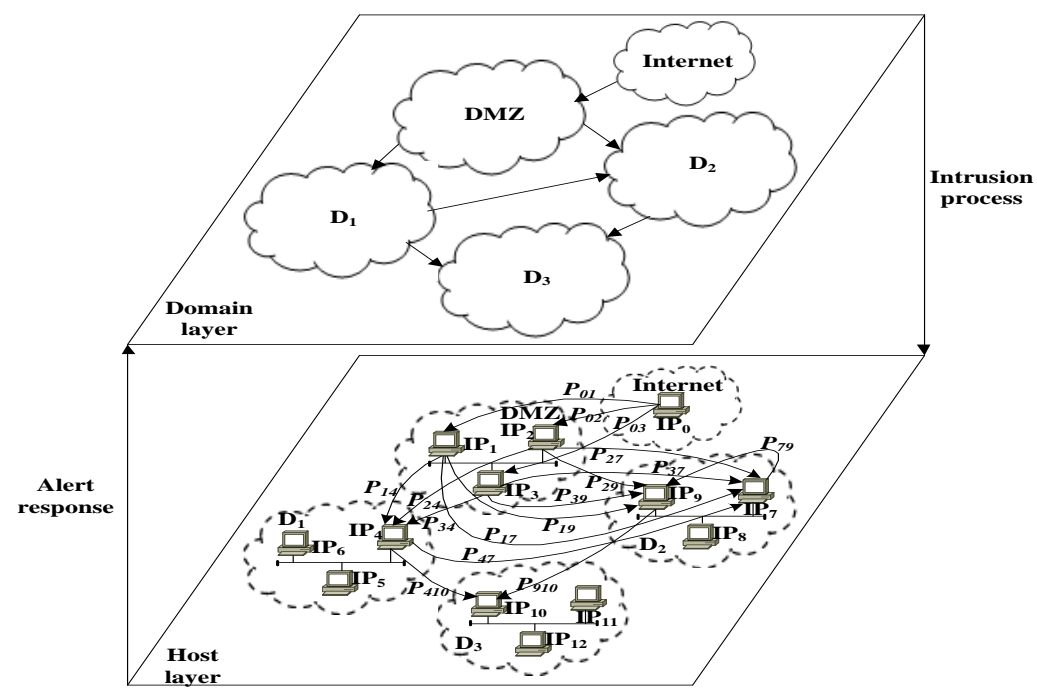

Figure 4. Domain-equipment Double Attack Graph of the Intrusion Intention $i$

In the double attack graph model shown in Figure 4, according to formula (1), the equipment $I P_{1}, I P_{2}, I P_{3}, I P_{4}, I P_{7}, I P_{9}$ and $I P_{10}$ intrusion probability can be calculated by administrators, there: $0.3,0.2,0.5,0.6,0.7,0.3$ and 0.5 , that in Figure 4, $\pi_{01}=0.3, \pi{ }_{02}=0.2$, $\pi_{03}=0.5, \pi_{14}=_{24}=\pi_{34}=0.6, \pi_{17}=\pi_{27}=\pi_{37}=\pi_{47}=0.7, \pi_{19}=\pi_{29}=\pi_{39}=\pi_{79}=0.3$, $\pi_{410}=\pi_{910}=0.5$. In the double attack graph model, there are total 12 kinds of intrusion route that achieve IPIO penetration for network equipment. Therefore, the probability of each route used by intruder is $1 / 12$, namely $\pi(r)=1 / 12 \approx 0.083$, each route and the probability distribution of the intrusion intention as shown in Table 2 . Among them, the probability $\pi$ represents the probability of each route is successfully invaded, according to the formula (2), the probability $i$ of intrusion intention can be derived, where: $\pi(i)=$ $1-(1-0.0900) \times(1-0.0189) \times \ldots \times(1-0.0750) \approx 0.4749$. 
According to the formula (3), it obtained the relative probability $\pi(r \mid i)$ for each intrusion route, as shown in Table 2, namely: $\pi\left(r_{1} \mid i\right)=\left(\pi_{1} \times \pi(r)\right) / \pi(i)=(0.0900 \times$ $0.083) / 0.4749 \approx 0.0157, \ldots$. In relative probability, $\pi\left(r_{9} \mid i\right)=0.0262$ is the maximum, $s_{o}$ the intruder is most likely to adopt this intrusion route $I P_{0} \rightarrow I P_{3} \rightarrow I P_{4} \rightarrow I P_{10}$ to achieve its intrusion intention. Strategy 2 can calculate the minimal right key set of each intrusion route in Table 2, where: $\left(I P_{4}, I P_{9}\right)$. To effectively prevent intruders achieve penetration intention $i$ of the network equipment $I P_{10}$, administrators can strengthen the protection for equipment $I P_{4}$ and $I P_{9}$, timely repairing related patches to restrict some users permission that access the database server $I P_{10}$. According to formula (4), we can calculate the cost of the maintenance of network security: $\operatorname{Cost}\left(I P_{6}\right)+\operatorname{Cost}\left(I P_{9}\right)$.

Table 2. Intrusion Route and the Probability of the Intention $i$

\begin{tabular}{cccc}
\hline$r$ & Intrusion route $(r)$ & Probability $\pi$ & Relative probability $\pi(r \mid i)$ \\
\hline 1 & $I P_{0} \rightarrow I P_{1} \rightarrow I P_{4} \rightarrow I P_{10}$ & 0.0900 & 0.0157 \\
2 & $I P_{0} \rightarrow I P_{1} \rightarrow I P_{4} \rightarrow I P_{7} \rightarrow I P_{9} \rightarrow I P_{10}$ & 0.0189 & 0.0033 \\
3 & $I P_{0} \rightarrow I P_{1} \rightarrow I P_{7} \rightarrow I P_{9} \rightarrow I P_{10}$ & 0.0315 & 0.0055 \\
4 & $I P_{0} \rightarrow I P_{1} \rightarrow I P_{9} \rightarrow I P_{10}$ & 0.0450 & 0.0079 \\
5 & $I P_{0} \rightarrow I P_{2} \rightarrow I P_{4} \rightarrow I P_{10}$ & 0.0600 & 0.0105 \\
6 & $I P_{0} \rightarrow I P_{2} \rightarrow I P_{4} \rightarrow I P_{7} \rightarrow I P_{9} \rightarrow I P_{10}$ & 0.0126 & 0.0022 \\
7 & $I P_{0} \rightarrow I P_{2} \rightarrow I P_{7} \rightarrow I P_{9} \rightarrow I P_{10}$ & 0.0210 & 0.0037 \\
8 & $I P_{0} \rightarrow I P_{2} \rightarrow I P_{9} \rightarrow I P_{10}$ & 0.0300 & 0.0052 \\
9 & $I P_{0} \rightarrow I P_{3} \rightarrow I P_{4} \rightarrow I P_{10}$ & 0.1500 & 0.0262 \\
10 & $I P_{0} \rightarrow I P_{3} \rightarrow I P_{4} \rightarrow I P_{7} \rightarrow I P_{9} \rightarrow I P_{10}$ & 0.0315 & 0.0055 \\
11 & $I P_{0} \rightarrow I P_{3} \rightarrow I P_{7} \rightarrow I P_{9} \rightarrow I P_{10}$ & 0.0525 & 0.0092 \\
12 & $I P_{0} \rightarrow I P_{3} \rightarrow I P_{9} \rightarrow I P_{10}$ & 0.0750 & 0.0131 \\
\hline
\end{tabular}

\section{Conclusion}

Intrusion intention identification as a means of network intrusion situation analysis and identification has become an important research direction in security management; it provides an important basis for managers to effectively determine the network vulnerabilities, providing some protection to prevent the occurrence of network intrusion. Thus, intrusion intention identification technology is one of the hotspot problems of today's network security research. On the previous research results ${ }^{[12]}$, the group optimized and presented a identification method that analysis intrusion intention automatically, detecting and making response based on the results of previous research which is domain-equipment double attack graph. The method refers the probability analysis means of Bayesian in the double attack graph, quantifying for each intrusion route in the figure, and determining the minimal right key set in network. Administrators can set the focused maintenance on the network equipment in the set, thus effectively preventing the implementation of intrusion intention. Next, the group will focus on the weaknesses layer to study, dividing the double attack graph into the domain-equipmentweaknesses attack graph. Quantifying intrusion route from the weakness layer will further improve the accuracy of determining intrusion intention, provides important data basis for more simple and efficient managing intranet.

\section{Acknowledgement}

Supported by National Natural Science Foundation of China (NO: 61502123) and Heilongjiang Provincial Youth Science Foundation (NO: QC2015084). 


\section{References}

[1] W. Peng, C.-Z. Hu, S.-P. Yao and Z.-G. Wang, “A Dynamic Intrusive Intention Recognition Method Based on Timed Automata", Journal of Computer Research and Development, (2011), vol. 48, no. 7, pp. 1288-1297.

[2] L.-X. Xiao, "Research on The Optimization of Enrollment Data Resources Based on Cloud Computing Platform", International Information and Engineering Technology Association, vol. 2, no. 2, (2015), pp. 9-12.

[3] L. Wang, "An Ungreedy Chinese Deterministic Dependency Parser Considering Long Distance Dependency”, International Information and Engineering Technology Association, vol. 2, no. 1, (2014), pp. 1-4.

[4] Bratman M. Intentions, Plans, and Practical Reason, Massachusetts: Harvard University Press, (1987).

[5] C. Phillips and L. Swiler, "A Graph-based System for Network Vulnerability Analysis", Proceedings of the New Security Paradigms Workshop, (1998); Charlottesville, VA, USA.

[6] O. Sheyner, J. Hainesand S. Jha, "Automated generation and analysis of attack graphs", Proc. IEEE Symposium on Security and Privacy, (2002); Oakland, California, USA.

[7] X. Ou, S. Govindavajhala and A. W. Appel, "MulVAL: A logic-based network security analyzer", Proceedings of the 14th Usenix Security Symposium, ACM, (2005); New York.

[8] R. Lippmann, K. Ingols and C. Scott, "Validating and Restoring Defense in Depth Using Attack Graphs", Proc. the Military communications Conference, IEEE Press, (2006); Washington, DC, USA.

[9] S. Roschke, F. Cheng and C. Meinel, "A new alert correlation algorithm based on attack graph", Proceedings of the 4th International Conference on Computational Intelligence in Security for Information Systems (CISIS), (2011).

[10] Y. Yun, X.-S. Xu, J. Yan, Z.-C. Qi, "An Attack Graph-Based Probabilistic Computing Approach of Network Security”, Chinese Journal of Computers, vol. 33, no. 10, (2010), pp. 1987-1996.

[11] X. J. Chen, B.-X. Fang, Q. F. Tan and H.-L. Zhang, "Inferring Attack Intent of Malicious Insider Based on Probabilistic Attack Graph Model”, Chinese Journal of Computers, vol. 37, no. 1, (2014), pp. 62-72.

[12] Z.-Y. Luo, G.-L. Sun, J.-H. Liu and W.-B. Wang, "Application of Attack Graphs Algorithms in Invasion Prevention System”, Journal of Yunnan University (Natural Sciences Edition), vol. 34, no. 3, (2012), pp. 271-275.

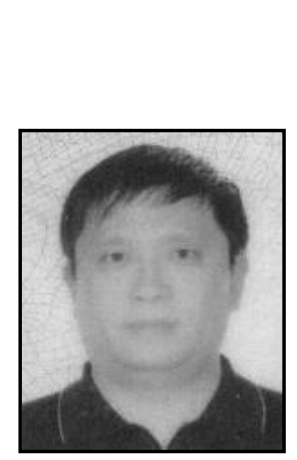

\section{Authors}

Wang Guangze, He received a bachelor's degree in Mechanical Engineering (1987) from the University. Now he is an associate researcher at the university library. His current research interests include different aspects of computer network technology and network security. 
International Journal of Security and Its Applications

Vol. 10, No. 3 (2016) 